\title{
An Interactive Content Development for Algorithm based Subjects in Engineering using Android
}

\author{
Prof. Sunita M. Dol ${ }^{1}$, Kaushal Kothari ${ }^{2}$, Sarthak Chankeshawara ${ }^{3}$, Pranav Gunde ${ }^{4}$ \\ Walchand Institute of Technology, Solapur ${ }^{1,2,3,4}$
}

\begin{abstract}
A software android application for algorithm based subjects in engineering features the visualization of commonly used algorithms in Data Structures and Design and Analysis of Algorithm. Various operations on stack, queue, list etc like insertion, deletion etc are introduced. In addition, this software can be used to animate various algorithms of searching, sorting, and operations on stack, queue etc. However, many students find difficulty to study the core subjects like Advanced C Concepts, Data Structure and Design \& Analysis of Algorithms because it requires abstract thinking. It would be very helpful if there will be visualization tool of data structures such as arrays, queues, stacks, trees and graphs for students to experiment with. The tool would allow students to see how an element is inserted into or deleted from different data structures, how a tree is traversed in different order (pre-order, in-order, post-order, level-order), etc. i.e. Simulation Technique. By using animation, it is possible to teach particular subject to the student in interesting \& easier way.
\end{abstract}

Keywords: Android, Eclipse, SDK (Software Development Kit), Advanced C Concepts, Data Structure, Design and Analysis of Algorithm, Simulation, Animation.

\section{INTRODUCTION}

Now a days, active learning strategies are needed to teach the students. Innovative techniques interactive content developments are also needed. In the present paper, an android application is proposed for Engineering subjects Advanced C concepts, Data Structure and Design and Analysis of Algorithm for giving better conceptual understanding of concepts of subject to students who feel these subjects difficulty while studying. Since these are the core subjects of Computer Science and Engineering having different complex algorithms and important for various competitive examination like GATE (Graduate Aptitude Test for Engineering), aptitude test of engineering, so we providing Simulation technique to understand the behavior of every technique related to the subject also the animation part makes learning interesting \& easier.

\section{LITERATURE REVIEW}

\section{Algorithms}

An algorithm is an app that allows easily viewing algorithm implementations. In this application how each algorithm can be implemented differently in different languages is given [1].

\section{Data Structures}

This feature-rich App contains C programs on various topics of Data Structures like stack, queue, linked list, trees, and graphs etc. which are frequently asked in college exams or job interviews [2].

\section{Programs}

This feature-rich App contains C Programs that are frequently asked in college exams or job interviews [3].

\section{Design \& Analysis of Algorithm}

This application is for all students of Design \& Analysis of Algorithms across the world. It covers 144 topics of Design \& Analysis of Algorithms in detail. These 144 topics are divided in 5 units [4].

\section{PROPOSED ARCHITECTURE OF APPLICATION}

The flow of this android based application is shown in figure 1. This software provides the information about following three subjects of Computer Science and Engineering.

\section{- Advanced C Concepts \\ Data Structure \\ Design and Analysis of Algorithm}

In this application, first user selects the subject. Depending on the subject selected, he will be able to read notes or execute the program or analyze the working of algorithm using animation. The flow of proposed application is shown in figure 1 . 


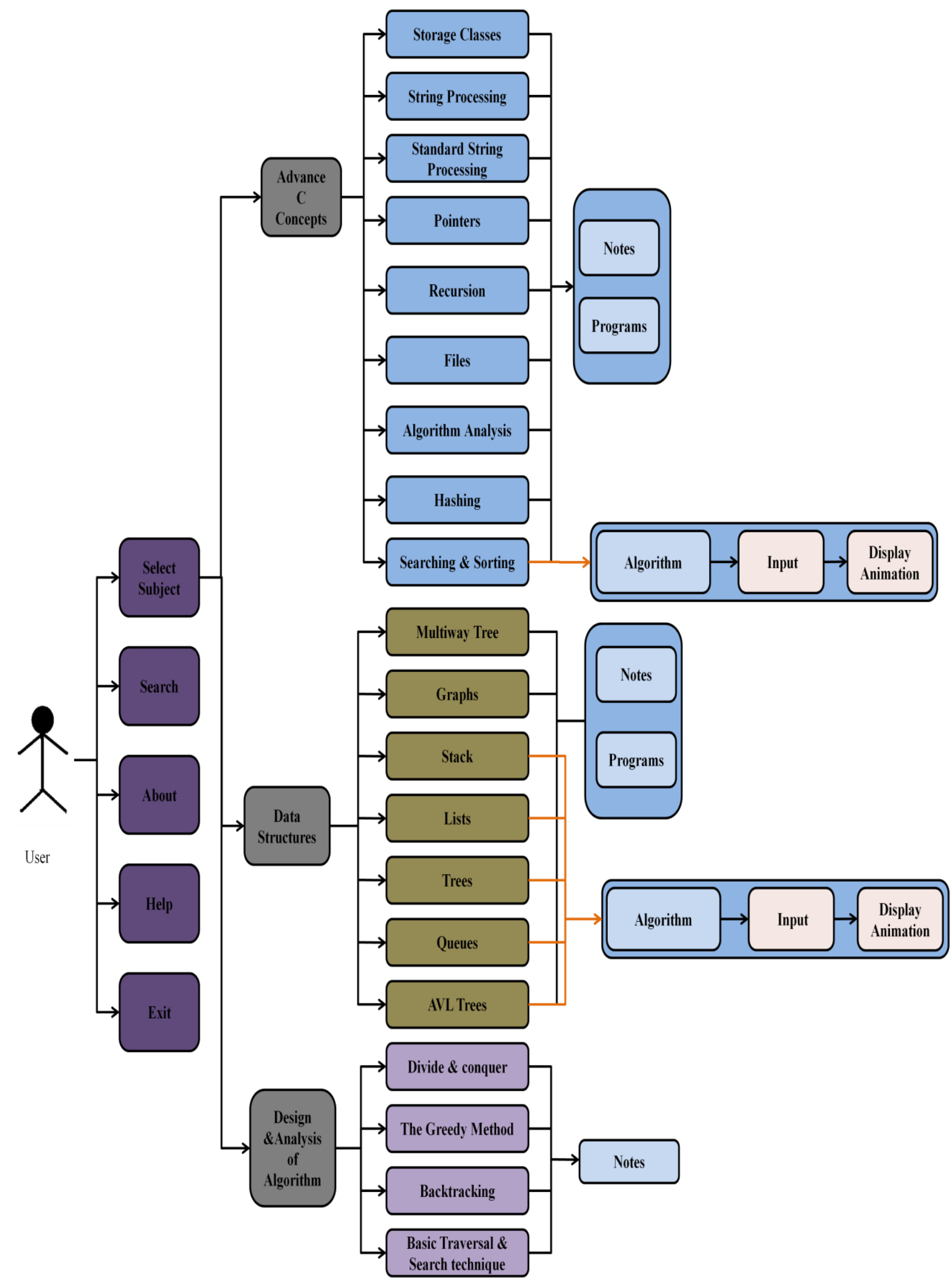

Figure 1: Flow of proposed application 


\section{TECHNOLOGY USED}

\subsection{Android SDK:}

Android software development is the process by which new applications are created for the Android operating system. Applications are usually developed in the Java programming language using the Android Software Development Kit, but other development tools are available.

Android SDK contains multiple files and tools specifically intended to design and develop applications that run on the Android platform. The bulk of the Android SDK, in number of files, consists of documentation, with programming APIs, tools, and samples comprising the rest. The SDK also includes help files, documentation, an Android Emulator, and a host of other development and debugging tools [6].

\subsection{Eclipse}

In computer programming, Eclipse is an integrated development environment (IDE) which contains a base workspace and an extensible plug-in system for customizing the environment. Eclipse is written mostly in Java, Eclipse can be used to develop applications. By means of various plug-ins, Eclipse may also be used to develop applications in other programming languages: Ada, ABAP, C, C++, COBOL, Fortran, Haskel 1, JavaScript, Lasso, Natural, Perl, PHP, Prolog, Python, R, Ruby(including RubyonRails framework), Scala, Clojure, Groovy, Scheme, and Erlang. Development environments include the Eclipse Java development tools (JDT) for Java and Scala, Eclipse CDT for $\mathrm{C} / \mathrm{C}++$ and Eclipse PDT for PHP, among others [5].

\section{CONCLUSION}

In this paper, the application useful for algorithm based subjects like Advanced C Concepts, Data Structure and Design and Analysis of Algorithm is proposed. This application will contain the study material, algorithms, programs and animation of algorithms.

\section{REFERENCES}

1. https://play.google.com/store/apps/details?id=com.kennyledet.algor ithms.app. grmeb\&hl=en

2. https://play.google.com/store/apps/details?id=com.datastructure \&hl =enhttp://groups.google.com/group/android-beginners

3. https://play.google.com/store/apps/details?id=com.spa\&hl=en

4. https://play.google.com/store/apps/details?id=com.faadooengineers. analysisalgorithm \&hl=en

5. http://en.wikipedia.org/wiki/Eclipse_(software)

6. http://en.wikipedia.org/wiki/Android_software_development 